\title{
Chylothorax secondary to late-onset congenital diaphragmatic hernia
}

\begin{abstract}
Late-onset congenital diaphragmatic hernia and chylothorax are rarely associated. We reported a 3-month-old male infant who presented with acute respiratory and gastrointestinal symptoms. Computed tomography of his chest showed features of diaphragmatic hernia with pleural effusion. Biochemistry charterers of pleural effusion identified chylothorax. The chylous effusion was drained and the diaphragmatic hernia was repaired by laparoscopic surgery. After surgery, the lung was fully expanded. Chylothorax was cured. In this case, lymphatic obstruction caused by the weight of the herniated bowel loops or by compression of lung may result in increased lymphatic pressure and contribute to the leakage of chyle into the pleural cavity.
\end{abstract}

Keywords: chylothorax, chylous effusion, diaphragmatic hernia, late onset, infant,child
Volume 2 Issue I - 2015

\section{Ching-Ying Huang,' Nan-Chang Chiu ${ }^{1,2}$ \\ 'Department of Pediatrics, MacKay Children's Hospital, Taiwan ${ }^{2}$ MacKay Junior College of Medicine Nursing and Management,} Taiwan

Correspondence: Ching-Ying Huang, Department of Pediatrics, MacKay Children $\square$ s Hospital, 92, Section 2, ChungShan North Road, Taipei, Taiwa, Tel +886-2-2543-3535, Fax +8862-2543-3642,Email b101090116@tmu.edu.tw

Received: December 15, 2014 | Published: January 14, 2015

\section{Introduction}

Congenital diaphragmatic hernia $(\mathrm{CDH})$ is a structural diaphragmatic defect that usually presents during the first few hours after birth with severe respiratory distress. However, delayed herniation of the abdominal contents through a CDH may occur beyond neonatal period. In contrast to the high mortality and morbidity rates for neonatal $\mathrm{CDH}$, the prognosis for late-presenting $\mathrm{CDH}$ is usually favorable if diagnosed earlier. However, unlike in neonates, the spectrum of manifestations of late-presenting $\mathrm{CDH}$ is quite broad and nonspecific. The patients may present with acute or chronic respiratory, gastrointestinal symptoms or be completely asymptomatic. ${ }^{1}$ The variety of clinical manifestations for late-onset $\mathrm{CDH}$ makes the early diagnosis challenging. Chylothorax, the accumulation of chyle in the pleural space, is a relatively rare cause of pleural effusion in children after neonatal period. ${ }^{2}$ Late-presenting $\mathrm{CDH}$ and chylothorax are rarely associated. We reported a 3-monthold infant of late-presenting $\mathrm{CDH}$ with development of chylous effusion.

\section{Case report}

A 3-month-old male infant brought to our hospital with a oneday history of poor appetite and activity. Gestational history was uneventful with birth weight 3520 grams and normal Apgar scores. He was healthy and had good weight gain before admission. The physical examination revealed severe respiratory distress, tachycardia, and acute ill looking. He had grunting, nasal flaring, and subcostal retraction. Air entry was reduced on the left side of the chest. His abdomen was flat with hypoactive bowel sounds. Chest radiograph showed opacification of left hemithorax with a moderate shift of mediastinum to right (Figure 1). He was admitted to the pediatric intensive care unit.

The hematologic and biochemical investigations were unremarkable except for leukocytosis $(18,800$ cells $/ \mu \mathrm{L})$. Clear yellowish fluid was drained from chest tube and the pleural fluid turned to milky white on the next day. Pleural fluid analysis revealed $\mathrm{pH} 7.5, \mathrm{LDH} 116 \mathrm{U} / \mathrm{L}$, protein $20.9 \mathrm{~g} / \mathrm{L}$, glucose $362 \mathrm{mg} / \mathrm{dL}$, total cholesterol $8 \mathrm{mg} / \mathrm{dL}$, triglyceride $391 \mathrm{mg} / \mathrm{dL}$, and predominance of lymphocytes, which of them were all compatible with chylous effusion. A computed tomography of the chest and abdomen showed left pleural effusion, lung atelectasis and retention of bowel loops in the anterior aspect of left hemi-thorax (Figure 2). The finding was consistent with diaphragmatic hernia.

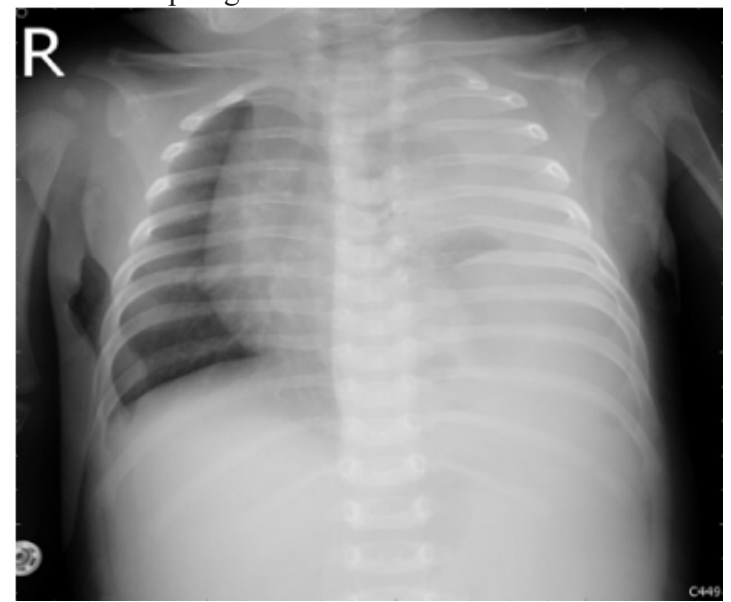

Figure I Initial chest x-ray showed left-sided opacification and a moderate shift of the mediastinum to the right.

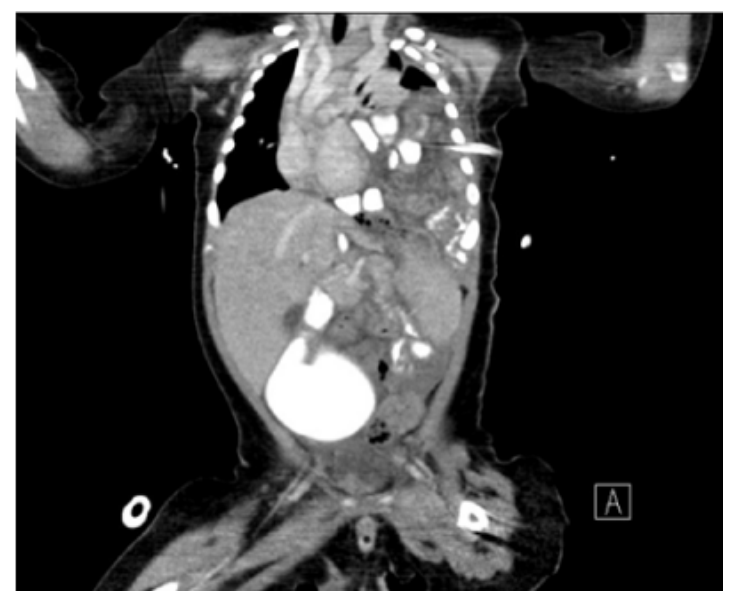

Figure 2 Chest CT demonstrated left diaphragmatic hernia and bowel loops, which contained barium contrast, in left thorax with pleural effusion and lung atelectasis. 
Surgical reduction was performed by laparoscope 2days later. During laparotomy, we found transverse colon and small bowel loops were herniated to left pleural cavity. A left posterior-lateral defect of diaphragm, measuring $3.5 \times 4 \mathrm{~cm} 2$, was repaired with absorbable mesh and fixed by screws. There was no perforation of bowel or thoracic duct. On the day following surgery, the patient was able to ingest formula, and was discharged 5 days postoperatively in good condition. The follow-up chest radiograph showed full expansion of lung and normal position of diaphragm.

\section{Discussion}

$\mathrm{CDH}$ is estimated to occur in 1 of 2,000-4,000 births. ${ }^{3}$ It is an embryonic malformation of the diaphragm occurring around the eight to ninth week of gestation and usually presents with respiratory distress soon after birth. ${ }^{4}$ However, some patients develop symptoms after the neonatal period. Late-presenting diaphragmatic hernia accounts 2.6$20 \%$ for all $\mathrm{CDH}$ and may first manifest at any age. ${ }^{5,6}$ The presentations of late-presenting $\mathrm{CDH}$ have wide variety including, in addition to respiratory distress, gastrointestinal obstruction, growth retardation, perforations or strangulations of intrathoracic hollow viscera, rupture of a herniated spleen, airway infections or recurrent pneumonias, urinary tract obstruction due to herniation of the ureter, intrathoracic appendicitis and other rare presentations. ${ }^{7,8}$ Chest radiography is usually the first diagnostic modality upon which further management is based. It is assumed that the presence of intrathoracic translucent structures along with mediastinal shift is the most characteristic radiological feature of late-presenting $\mathrm{CDH}$, but only when hernia contains stomach or bowel. Misinterpretation of initial radiographic findings occurred in more than $25 \%$ of the children and nearly $50 \%$ of the children require additional imaging studies to achieve a final diagnose. ${ }^{9}$ Our patient is a 3-month-old infant presenting with acute respiratory and gastrointestinal symptoms. Chest x-ray revealed opacification of left hemithorax with shifted mediastina. Further computed tomography made a final diagnosis of CDH. Under prompt detection, the patient's herniation of bowel loops was reduced and the affecting lung was fully expanded after surgery. His follow-up condition is well 3 months later.

The association of chylothorax characterizes this patient. From review of the literature, we did not found previously reported case of an infant with late-presenting $\mathrm{CDH}$ causing chylous effusion. Chylothorax is a pleural collection of fluid formed by the escape of chyle from the thoracic duct or lymphatics into the thoracic cavity. It is the most common form of pleural effusion in neonate but is a rare cause of pleural effusion in infants and children., ${ }^{2,10}$ Chyle is a non-inflammatory, alkaline and bacteriostatic fluid composed mainly of fat, cholesterol, electrolytes, proteins, glucose, and abundant lymphocytes. The protein content of chyle is usually more than $3 \mathrm{~g} / \mathrm{L}$, and the electrolyte composition is similar to that of serum. The cells are primarily T lymphocytes. ${ }^{11}$ Measuring the triglyceride and cholesterol levels in the pleural fluid is the best way to diagnose chylothorax. If the triglyceride is above $110 \mathrm{mg} / \mathrm{dL}$ and the ratio of the pleural fluid to serum cholesterol is less than 1.0, the diagnosis is established..$^{12}$ In our case, the milky fluid from the patient showed marked triglyceride elevation and low ratio of pleural fluid to serum cholesterol, which were compatible with chylothorax.

In normal physiology, the gut lymphatics carry converted longchain triglycerides from the intestine to the vascular system via the thoracic collecting duct, which transports 1.5 to 2.5 liters of chyle daily in children. ${ }^{2}$ As a result, in cases of acute lymphatic obstruction, rapid accumulation of chyle can occur. In children, development of chylothorax is more commonly associated with cardiothoracic surgery with an incidence of $65-90 \% \cdot{ }^{13,14}$ Other important causes in children include congenital lymphatic malformations, associated with congenital anomaly, non-iatrogenic trauma, and tumors. ${ }^{15}$ In our patient, we postulate that the herniation of intestinal loops in the thorax had led obstruction of lymphatic flow from the weight of the bowel loops or compression of lung. The increasing lymphatic pressure contributed to the leakage of chyle, which eventually accumulated in the pleural cavity.

The optimal management of chylothorax depends on its underlying etiology. Imaging studies such as computed tomography, lymphangiography, and lymphoscintigraphy can be helpful to determine the cause of the chylothorax. ${ }^{2}$ In our case, thoracic duct injury by chest tube was considered but less likely since this procedure was echo-guided and the operation performed later did not find evidence of thoracic duct damage. The patient's chylothorax also totally resolved after the surgery. Hence, the cause of the chylous effusion could be confidently attributed to herniation of bowel loops, which resulted in temporary lymphatic obstruction.

\section{Acknowledgements}

We would like to acknowledge Dr. Nien-Lu, Wang for his assistance with performing the surgery.

\section{Conflicts of interest}

Author declares there are no conflicts of interest.

\section{Funding}

None.

\section{References}

1. Baerg J, Kanthimathinathan V, Gollin G. Late-presenting congenital diaphragmatic hernia: diagnostic pitfalls and outcome. Hernia. 2012;16(4): 461-466.

2. Soto-Martinez M, Massie J. Chylothorax: diagnosis and management in children. Paediatr Respir Rev. 2009;10(4):199-207.

3. Doyle NM, Lally KP. The CDH Study Group and advances in the clinical care of the patient with congenital diaphragmatic hernia. Semin Perinatol. 2004;28(3): 174-184.

4. Vukic Z. Left Bochdalek hernia with delayed presentation: report of two cases. Croat Med J. 2001;42(5):569-571.

5. Kitano Y, Lally KP, Lally PA. Late-presenting congenital diaphragmatic hernia. J Pediatr Surg. 2005;40(12):1839-1843.

6. Chang SW, Lee HC, Yeung CY, et al. A twenty-year review of early and late-presenting congenital Bochdalek diaphragmatic hernia: are they different clinical spectra? Pediatr Neonatol. 2010;51(1):26-30.

7. Osebold WR, Soper RT. Congenital posterolateral diaphragmatic hernia past infancy. Am J Surg . 1976;131(6):748-754.

8. Amirav I, Kramer SS, Schramm CM. Radiological cases of the month. Delayed presentation of congenital diaphragmatic hernia. Arch Pediatr Adolesc Med. 1994;148(2):203-204.

9. Baglaj M, Dorobisz U. Late-presenting congenital diaphragmatic hernia in children: a literature review. Pediatr Radiol . 2005;35(5):478-488.

10. van Straaten HL, Gerards LJ, Krediet TG. Chylothorax in the neonatal period. Eur J Pediatr. 1993;152(1):2-5.

11. Teba L, Dedhia HV, Bowen R, et al. Chylothorax review. Crit Care Med. $1985 ; 13(1): 49-52$. 
12. Light RW. Chylothorax and pseudochylothorax. In: Wolters Kluwer (Ed.), Pleural Diseases. (6th edn), Lippincott Williams and Wilkins, Philadelphia, USA. 2013. p. 412-430.

13. Doerr CH, Allen MS, Nichols FC, et al. Etiology of chylothorax in 203 patients. Mayo Clin Proc . 2005;80(7):867-870.
14. Haines C, Walsh B, Fletcher M, et al. Chylothorax development in infants and children in the UK. Arch Dis Child. 2014;99(8): 724-730.

15. Tutor JD. Chylothorax in infants and children. Pediatrics. 2014;133(4):722-733 